\title{
COMMENTS
}

\section{Promoting Agriculture Green Development to realize the great rejuvenation of the Chinese nation}

\author{
Xinrong YU (凶) \\ Ministry of Agriculture and Rural Affairs of the People's Republic of China, Beijing 100125, China
}

With the development of the new century, China's agricultural modernization has reached a critical juncture. It has become a matter of extreme urgency to determine which of a number of possible paths should be taken. The traditional way of agricultural production and development in China (and many other countries) often addresses only single, isolated problems of food production in the whole industry chain. The traditional way and its concepts, technologies, and standards are obsolete and no longer sustainable. It is a necessity now to seek a balance between stabilizing agricultural production and protecting the natural environment. A fundamental pathway which allows us to move towards this target is Agriculture Green Development (AGD).

China Agricultural University has created the National Academy of Agriculture Green Development to organize scientific and technological forces to devote themselves to multidisciplinary and comprehensive research on AGD. This is not only fundamental and comprehensive scientific and technological innovation work, but also a concrete action plan to implement the Central Government's major strategic measures to support AGD and promote the Government's eco-civilization construction. At the present time, China's agricultural modernization needs to cross many thresholds. It is undoubtedly an urgent, arduous and long-term mission to explore the paths of agricultural modernization for China. The paths taken by the world's agricultural systems as they are modernized to address growing problems associated with food, farming, the natural environment and human health are also relevant to China.

Agricultural production and the supply of safe healthy food are closely intertwined with economic, environmental, and policy development in many countries. Presently, agricultural development in China and more generally around the globe is confronted with highly significant problems and challenges. One of the key issues is the rise in frequency and severity of ecological catastrophes, for example, which recently resulted in the devastation of tropical rainforests in Central America, also indeed related to weather extremes, political pressures, economic development, etc. In China, pork prices have risen sharply in 2019 due to factors linked to African swine fever, as well as restrictions on raising pigs to limit damage to the environment because of administrative and managerial controls put in place for precautionary measures in pig farming. In some places, intensive agricultural production can be highly damaging to the natural ecological environment, and problem-solving attempts are oftentimes designed in a singular or linear manner, and as such, a theoretical basis for development of systemic solutions is lacking. To fundamentally solve these problems, we need to deepen the theory and practice of AGD, and formulate green solutions to address the new challenges facing agriculture, rural development and farmers' wellbeing worldwide.

Some countries in Central America have asked for China's assistance in areas such as coffee planting, as the cultivation of coffee plants by farmers on the hillsides not only increases the income of farmers, but also avoids the felling of hillside trees, thus protecting the local ecological environment. Some countries in Europe are developing organic agriculture, but the proportion of land used for organic agriculture is relatively low, as it erodes the income of farmers, to some extent. Also, the extensive approach to relatively low-productivity food production requires more land in circumstances where most good-quality land is already in production. China and much of the rest of the world

Received January 3, 2020

Correspondence: xxrr6699@126.com

(C) The Author(s) 2020. Published by Higher Education Press. This is an open access article under the CC BY license (http://creativecommons.org/licenses/by/4.0) 
have encountered situations that involve sacrificing the environment to protect agriculture, or vice versa. Neither approach is satisfactory; both approaches are problematic. Traditional production and development in terms of modes, concepts, technologies, and standards are often no longer sustainable; they cannot fulfill the requirements of the new era. In particular, they cannot provide solutions to the food security and development challenges of 1.4 billion people in China, nor can they address the major survival and development issues for the estimated 10 billion people who will populate the world by 2050.

AGD involves a deep revolution in development concepts, ideas, and technologies. It requires innovative thinking and integration from multiple sectors and particularly by the whole-industry chain in agriculture. More critically, it requires innovation in science and technology, agricultural education system and social service models. China will pool the efforts of all sectors to deepen research.

Concepts determine ideas, and ideas determine pathways. It is crucial to understand what principles should be followed in considering AGD, and what new pathways should be opened up? The development of agriculture in China and the world has ushered in a great period of change in the past millennium. We must push on and advance forward; retreat is not an option.

AGD is a breakthrough of ideas and concepts, a profound revolution in the developmental concept of agriculture, and a technological revolution. It is closely related to the great rejuvenation of the Chinese nation. Green development will require novel technological approaches, financial and institutional support and will also need continuing theoretical innovation of AGD concepts. Moreover, AGD requires theory simplification, technological modernization, evaluation indexation, and measurement instrumentation, to support the continued prosperity and progress of future societies. The development of the concepts on green development in China is already having a profound impact on agricultural development in the rest of the world.

There is a long way to go in terms of scientific research. We need to continuously deepen research and promote Government-Industry-University-Research-Application collaborative innovation, to benefit 260 million farmers across the country and all Chinese citizens, and truly realize a profound revolution in the development concept of agriculture. We look forward to promoting the green development of China's agriculture on the basis of China's agricultural culture and traditions, as well as to proposing effective developments that can offer guidance and provide a framework for sustainable development of the world's agriculture.

Acknowledgements This commentary is a translation of Chinese script, translated by Prof. Jianbo Shen (China Agricultural University, China) and Prof. William J. Davies (Lancaster University, UK), and revised by Prof. Zhengxia Dou (University of Pennsylvania, USA). 